\title{
Research Article
}

\section{Stroke mortality: predictive value of simple laboratory tests and acute physiology, age, chronic health evaluation III scoring system: a hospital based study}

\author{
Ajeet K. Chaurasia*, Manoj K. Mathur, N.C. Dwivedi, Manjul Mishra
}

Department of Medicine, MLN Medical College, Allahabad, Uttar Pradesh, India

Received: 24 February 2016

Accepted: 22 March 2016

\section{*Correspondence:}

Dr. Ajeet K. Chaurasia,

E-mail: ajeetkc30@gmail.com

Copyright: (C) the author(s), publisher and licensee Medip Academy. This is an open-access article distributed under the terms of the Creative Commons Attribution Non-Commercial License, which permits unrestricted non-commercial use, distribution, and reproduction in any medium, provided the original work is properly cited.

\begin{abstract}
Background: Acute stroke is a heterogeneous condition with respect to prognosis. This study was undertaken with the aim to evaluate the significance of routine simple blood parameters and APACHE (acute physiology, age, chronic health evaluation) III scoring system as methods of prediction of 1-month mortality in stroke patients and to assess the sensitivity and specificity of APACHE III scoring system in predicting short term outcome in critically ill patients having stroke.

Methods: Patients of stroke presenting within 48 hours of onset were included in the study irrespective of age, sex or type of stroke. The clinical evaluation, neuroimaging and blood investigations were performed. APACHE III scoring system was applied for each patient to calculate the score for each one. The outcome of the patients at the end of one month was determined as survivors and expired.

Results: Among the total 120 cases in the study, 54.16\% $(n=65)$ patients survived at the end of one month. The mean APAPCHE III Score among the survivors was 45.3 and in expired patients it was 88.6. Taking a cut-off value of 50, APACHE III Score was significantly associated in predicting the mortality in stroke patients $(\mathrm{P}$-value $<0.0001)$.

Conclusions: The study concluded that a low GCS at the time of admission and increased serum creatinine were independent predictors of mortality among patients presenting to the hospital within 48 hours with first time acute stroke. APACHE III scoring system was found to be sensitive and reasonably specific in predicting short term outcome in patients having cerebral stroke.
\end{abstract}

Keywords: Stroke, APACHE III, CVA

\section{INTRODUCTION}

Stroke defines an acute vascular event in the brain and is a leading cause of death and disability worldwide. Stroke ranks first in frequency and importance among all neurological disorders. At least $50 \%$ of neurological disorders are of this type. Stroke is uncommon in younger age groups and affects males more often than females. ${ }^{1-6}$

The diagnosis of a stroke is often clinical aided by neuroimaging. A stroke is a medical emergency and can cause permanent neurological damage, complications and death. Risk factors of stroke are both modifiable and nonmodifiable. Results of stroke vary widely depending on the size and location of lesions.

The chances of survival in stroke depend on a great variety of variables.

Factors associated with poor outcome after strokes are as following. 
- Demographic features: Increasing age

- Clinical features: (a) general: fever, urinary incontinence, previous stroke, atrial fibrillation, cardiac failure, ischemic heart disease, diabetes mellitus; (b) neurological: reduced levels of consciousness, severe motor deficit, impaired proprioception, visuospatial dysfunction, cognitive impairment.

- Simple laboratory tests: hyperglycemia, high hematocrit.

- CT and MRI: large lesion, mass effect, intraventricular bleeding, hydrocephalus.

Acute stroke is a heterogeneous condition with respect to prognosis. It is not possible to predict exact outcome of stroke with accuracy. Various studies have been undertaken to assess the prognosis of both ischemic and hemorrhagic stroke evaluating different clinical and biochemical parameters.

This study was undertaken with the aim to evaluate the significance of routine simple blood parameters and APACHE III scoring system as methods of prediction of 1-month mortality in stroke patients and to assess the sensitivity and specificity of APACHE III scoring system in predicting short term outcome in critically ill patients having stroke.

\section{METHODS}

The proposed study was conducted in the Department of Medicine, S.R.N. Hospital, Allahabad, India during a period of one year from July 2011 to July 2012.

All patients of stroke presenting to the medicine department within 48 hours of onset were included in the study irrespective of age, sex or type of stroke. Patients presenting to the hospital 48 hours after the onset of the signs and symptoms of stroke, patients with prior stroke, patients with intracerebral hemorrhage due to bleeding from brain tumor and hemorrhagic transformation of a cerebral infarct were excluded from the study.

The clinical evaluation, neuroimaging and blood investigations were performed. APACHE III scoring system was applied for each patient to calculate the score for each one. APACHE III scoring system consists of the sum of scores [0-299] from evaluation of the following:

- Vital signs \& Laboratory results [0-175]

- Neurological score [0-77]

- Age score [0-24]

- Chronic health score [0-23]

The patients were followed up for a maximum period of 1 month from the onset of stroke. The outcome of the patients at the end of one month was determined as survivors and expired. Logistic regression analysis was carried out among the significant parameters to identify independent predictors of 1-month fatality. APACHE III score for each patient was correlated with the outcome within the first month.

\section{RESULTS}

Among the total 120 cases in the study, 54.16\% $(n=65)$ patients survived at the end of one month and $45.84 \%$ $(n=55)$ patients expired during the hospital stay. $49.16 \%$ $(\mathrm{n}=59)$ patients suffered from ischemic stroke and $50.83 \%(n=61)$ number suffered from hemorrhagic stroke with one patient having subarachnoid hemorrhage.

Among the patients who expired the mean age were 68.3 years and 64.7 years in the survived patients. Maximum number of cases $(n=49)$ were in the age group 61-70 years. The incidence of stroke was higher in male patients. Out of total 120 patients, $60 \%(n=72)$ were male and $40 \%(n=48)$ were female. Among these $48.6 \%$ of male patients $(n=35)$ suffered from hemorrhagic stroke and $51.4 \%(n=37)$ suffered from ischemic stroke. $54.16 \%$ of female patients $(n=26)$ suffered from hemorrhagic stroke and $45.84 \%(n=22)$ had ischemic stroke.

Hypertension was the most common risk factor in our study present in $88.3 \%(n=106)$ patients, among which it was diagnosed for the first time in $72.6 \%(n=77)$ patients. Diabetes mellitus was present in $24.16 \%(n=29)$ patients. Hypertension and diabetes mellitus co-existed in $22.5 \%$ $(n=27)$ patients. Two patients $(n=2)$ had rheumatic heart disease (mitral stenosis). One patient $(n=1)$ suffered from cirrhosis of liver.

In our study, there was no statistical significance in terms of outcome with respect to pulse rate, respiratory rate, temperature, hemoglobin, TLC, ESR, serum electrolytes, serum albumin, serum bilirubin, serum ALT and serum LDL levels.

The mean APACHE III score calculated in the study was 65.08 \pm 29.13 . The highest APAPCHE III score calculated in the study was 127 . The lowest APAPCHE III score in the study was 25 .

The mean APAPCHE III Score among the survivors was 45.3 and the mean APACHE III Score among the expired patients was 88.6. The mean APACHE III Score in the patients of ischemic stroke was $56.47 \pm 28.73$, whereas the mean APACHE III Score in patients of hemorrhagic stroke was $73.4 \pm 29.13$.

Taking a cut-off of APACHE III Score of 50 and applying Chi Square test in hemorrhagic stroke, the Chi Square value came out to be 34.6 (P-Value $<0.0001)$. 
Table 1: Distribution of parameters (physiological and laboratory tests) in relation to stroke outcome (survivors/expired).

\begin{tabular}{|c|c|c|c|}
\hline \multirow{2}{*}{ Parameters } & \multicolumn{2}{|l|}{ Outcome } & \multirow{2}{*}{ P-value } \\
\hline & Survivors (n=65) & Expired $(\mathrm{n}=55)$ & \\
\hline GCS & $11.09 \pm 2.88$ & $5.4 \pm 3.016$ & $0.0000(\mathrm{~S})$ \\
\hline Pulse (per min) & $82.707 \pm 8.272$ & $84.296 \pm 12.705$ & $0.4100(\mathrm{~N})$ \\
\hline Mean BP (mmHg) & $116.095 \pm 17.399$ & $124.86 \pm 26.262$ & $0.0200(\mathrm{~S})$ \\
\hline $\operatorname{Temp}\left({ }^{\circ} \mathrm{C}\right)$ & $37.703 \pm 0.467$ & $37.741 \pm 0.479$ & $0.6554(\mathrm{~N})$ \\
\hline Resp rate(per min) & $18.676 \pm 3.779$ & $19.236 \pm 3.819$ & $0.4230(\mathrm{~N})$ \\
\hline $\mathrm{PaO}_{20}$ & $92.569 \pm 4.585$ & $92.909 \pm 4.762$ & $0.6918(\mathrm{~N})$ \\
\hline Hematocrit (\%) & $32.307 \pm 4.316$ & $33.627 \pm 6.254$ & $0.1779(\mathrm{~N})$ \\
\hline TLC (cells per cumm) & $12118.46 \pm 3422.576$ & $13467.5 \pm 5713.77$ & $0.1132(\mathrm{~N})$ \\
\hline S.creatinine (mg\%) & $0.98 \pm 0.335$ & $1.672 \pm 0.841$ & $0.0000(\mathrm{~S})$ \\
\hline Urine Output (mL) & $1738.462 \pm 506.116$ & $936.364 \pm 399.705$ & $0.0000(\mathrm{~S})$ \\
\hline BUN (mg\%) & $18.212 \pm 5.036$ & $31.043 \pm 16.951$ & $0.0000(\mathrm{~S})$ \\
\hline S.Albumin (g/dL) & $3.475 \pm 0.406$ & $3.666 \pm 0.596$ & $0.1802(\mathrm{~N})$ \\
\hline S.Bilirubin (mg\%) & $1.003 \pm 0.389$ & $1.076 \pm 0.671$ & $0.4513(\mathrm{~N})$ \\
\hline AST (IU/L) & $46.261 \pm 29.997$ & $73.11 \pm 88.09$ & $0.0229(\mathrm{~S})$ \\
\hline ALT (IU/L) & $40.748 \pm 10.209$ & $47.938 \pm 48.44$ & $0.2454(\mathrm{~N})$ \\
\hline RBS (mg\%) & $129.818 \pm 69.851$ & $193.609 \pm 79.435$ & $0.0000(\mathrm{~S})$ \\
\hline ESR (mm) & $24.276 \pm 5.461$ & $25.618 \pm 8.945$ & $0.3157(\mathrm{~N})$ \\
\hline Length of stay (days) & $6.6 \pm 3.811$ & $4.09 \pm 2.73$ & $0.0001(\mathrm{~S})$ \\
\hline
\end{tabular}

Table 2: APACHE III score in hemorrhagic stroke.

\begin{tabular}{|llll|}
\hline APACHE III Score & Death & Survival & Total \\
\hline$<50$ & 2 & 18 & 20 \\
\hline$\geq 50$ & 36 & 5 & 41 \\
\hline Total & 38 & 23 & 61 \\
\hline
\end{tabular}

Taking a cut-off of APACHE III Score of 50 and applying Chi Square test in ischemic stroke, the Chi Square value came out to be $24.35(\mathrm{P}-$ Value $<0.0001)$.

Table 3: APACHE III score in ischemic stroke.

\begin{tabular}{|llll|}
\hline APACHE III Score & Death & Survival & Total \\
\hline$<50$ & 2 & 34 & 36 \\
\hline$\geq 50$ & 15 & 8 & 23 \\
\hline Total & 17 & 42 & 59 \\
\hline
\end{tabular}

Table 4: Data obtained after applying multivariate logistic regression on significant parameters.

\begin{tabular}{|lllll|}
\hline & B & S.E. & Sig. & Exp(B) \\
\hline RBS & .006 & .004 & .112 & 1.007 \\
\hline Mean BP & .001 & .015 & .946 & 1.001 \\
\hline S. Creatinine & 2.334 & .638 & .000 & 10.323 \\
\hline AST & .010 & .008 & .193 & 1.010 \\
\hline Length of Stay & -.090 & .104 & .387 & .914 \\
\hline \hline Glasgow Coma Sca & $\mathbf{- . 4 3 3}$ & $\mathbf{. 1 0 4}$ & $\mathbf{. 0 0 0}$ & $\mathbf{. 6 4 8}$ \\
\hline
\end{tabular}

B = Coefficient; S.E. = Standard error; Sig = Significance in the particular test; $\operatorname{Exp}(B)=$ Expression of odds.
Multivariate logistic regression analysis was performed among the significant parameters in the undertaken study. The data obtained from the analysis was as shown in table.

\section{DISCUSSION}

In our study, maximum number of cases $(n=49)$ were in the age group 61-70 years. Vermeer $\mathrm{S}$ et al in 2002 found that age of 65 years or more doubled the risk of occurrence of stroke. ${ }^{4}$ Similar observation was made by Ong TZ et al in 2002 in their study on stroke patients where the mean age of the stroke patients was 65 years. $^{7}$

The total number of male and female patients in the study was $72(60 \%)$ and $48(40 \%)$ respectively. Among these $48.6 \%$ of male patients $(n=35)$ suffered from hemorrhagic stroke and $51.4 \%(n=37)$ suffered from ischemic stroke. This was in correlation with the previous studies by Sacco RD et al in $1998 .^{8}$ Appelros P et al in a Systematic Review in 2009 found that the incidence rates of intracerebral hemorrhage were higher among men. ${ }^{3}$

The mean BP was higher among the expired patients which was statistically significant $(\mathrm{P}$-value $<0.05)$. Similar observation was made by $\mathrm{T}$ Zong et al in 2002 and Smajlović D et al in 2008 where hypertension was found to be significant predictor of one month mortality. ${ }^{7,8}$ Sami Tetri et al in 2009, in a prospective study found that admission MABP was higher in those who died in two days $(\mathrm{P}-$ Value $<0.001)$ and within three months (P- 
Value $<0.01)$ than those who survived. High admission MABP predicted early death among the patients. Maria Sessa et al in 2008 studied relationship between hypertension and $\mathrm{ICH}$ in a systematic review on risk factor for ICH in general population. Of the 11 casecontrol and 3 cohort studies identified in the different searches for studies on hypertension as risk factor for $\mathrm{ICH}$, all showed a positive association between hypertension and $\mathrm{ICH}^{9,10}$

The mean temperature of the patients in our study was statistically insignificant among the survivors and expired ( $P$ value $>0.05$ ). Szczudlik A et al in 2002 concluded that patients with hyperthermia on the first day of hospitalization have a greater 30-day mortality and worse functional status 30 days after stroke, but increased body temperature is not an independent predictor of 30-day mortality after primary intracerebral hemorrhage. ${ }^{11}$

The mean RBS in the expired patients in our study was $193.6 \pm 79.4 \mathrm{mg} \%$. Edwin et al in 2006 demonstrated that hyperglycemia predicts an increased risk of in hospital mortality after ischemic stroke $(\mathrm{RR}=3.3) .{ }^{12} \mathrm{Hitt} \mathrm{E}$ et al in a population-based study in 2007 suggested that patients with diabetes mellitus appear to be at a 3 to 4 fold increased risk of intracerebral hemorrhage (ICH) compared with patients without DM. ${ }^{13}$ Schlenk et al in 2009 showed that blood glucose levels greater than 7.8 $\mathrm{mmol} / \mathrm{l}$ at the time of admission independently predicted unfavourable outcome. ${ }^{14}$ Feng-zeng li et al in showed that hyperglycemia was an independent predictor of mortality within 30 days (AUC-0.718). ${ }^{15}$

In our study the mean serum creatinine of the expired patients was statistically significant. Similar observation was made by Sweileh-Weim et al in 2008 where increased serum creatinine was found significant $(\mathrm{P}$ value $=0.004$ ) in predicting stroke mortality. ${ }^{16}$

The mean GCS of the expired patients at the time of admission was 5.4 \pm 3.01 . Similar observations were made by TZ Ong et al in 2002 where GCS <9 was an independent predictor of mortality. ${ }^{7}$ Weir $\mathrm{CJ}$ et al in their study in 2003 concluded that total GCS score predicted acute mortality with $88 \%$ accuracy. ${ }^{17}$ In a study by Md. Mahbub S et al in 2009, a GCS less than 8 at the time of admission was found to be an independent predictor of mortality in patients of acute stroke. ${ }^{18}$

Increased BUN and decreased urine output were statistically significant in the expired group of patients. Similar observations were made by Wafik et al in 2009in their study on patients with first time acute stroke. ${ }^{19}$ The mean AST level in the expired group of patients under study was statistically significant. Similar observation was made by Kim et al in 2005 where AST level $>70 \mathrm{IU} / \mathrm{L}$ was identified as a predictor of stroke mortality. ${ }^{20}$
Sico JJ et al in 2012 observed that a very low hematocrit $(<27 \%)$ was associated with early post-stroke mortality. ${ }^{21}$ Even a moderate level of anemia was independently associated with an increased risk of death during the first year following acute ischemic stroke. In our study we did not observe any prognostic value of hematocrit measurement at the the time of admission. This could be explained by the fact that the mean hematocrit in our patients was $32.91 \pm 5.33 \%$.

In our study we did not observe any prognostic value of electrolyte measurements at the the time of admission. Similar observations were made by Woo et al in 1990 in their study on stroke patients. ${ }^{22}$

Most of the scoring systems, used to determine short term outcome in stroke, consider only the neurological deficit related parameters when evaluating these patients while APACHE III scoring system takes into consideration various other parameters in addition to the neurological deficit. Thus, it may be a better alternative and a good, effective predictor of short-term outcome in cerebrovascular accident patients. In the present study we observed that the likelihood of mortality increased as the score increased and the most noticeable increase was seen when the score was more than 50.The sensitivity of this score at a cutoff point of 50 was $88.2 \%$ for ischemic stroke and $94.7 \%$ for hemorrhagic stroke. However, the specificity of the score was $80.9 \%$ for ischemic stroke and $78.2 \%$ for hemorrhagic stroke. In a previous study done by Bhalla et al in 2002, the sensitivity and specificity of APACHE III scoring system in predicting mortality was $94.12 \%$ and $53.85 \%$ respectively, in patients with hemorrhagic stroke and $90 \%$ and $73.53 \%$ respectively for ischemic stroke when a cutoff point of 40 was taken. ${ }^{23}$ In a study done by Wafik et al in 2009, sensitivity and specificity of APACHE III scoring system in predicting mortality was $88 \%$ and $74 \%$, respectively, in patients with hemorrhagic stroke and $89 \%$ and $70 \%$ respectively for ischemic stroke when a cutoff point of 40 was taken. ${ }^{19}$ The sensitivity and the specificity of the present study correlated with the previous ones.

\section{CONCLUSION}

It was observed after analysis that a low GCS at the time of admission and increased serum creatinine were independent predictors of mortality among patients presenting to the hospital within 48 hours with first time acute stroke. APACHE III scoring system, which uses simple measures and can be applied easily in every patient, was found to be sensitive and reasonably specific in predicting short term outcome in critically ill patients having cerebral stroke.

Funding: No funding sources

Conflict of interest: None declared

Ethical approval: The study was approved by the Institutional Ethics Committee 


\section{REFERENCES}

1. Sacco RL, Adams R, Albers G, Alberts MJ, Benavente O, Furie K, et al. Guidelines for prevention of stroke in patients with ischemic stroke or transient ischemic attack. Stroke, 2006;37:577-98.

2. Sacco RL, Benjamin EJ, Broderick JP. American Heart Association Prevention Conference IV: Prevention and rehabilitation of stroke: risk factors. Stroke 1997;28:1507-17.

3. Sex Differences in Stroke Epidemiology: A Systematic Review Peter Appelros, Birgitta Stegmayr and Andreas Terént. Stroke. 2009,40:108290.

4. Vermeer SE, Algra A, Franke CL, Koudstaal PJ, Rinkel GJE. Long-term prognosis after recovery from primary intracerebral haemorrhage. Neurology. 2002;59(2):205-9.

5. Smith S, English JD, Johnston SC. Cerebrovascular diseases. Harrison's Principles of Internal Medicine. 2011;18(370):3270-99.

6. Victor A. Cerebrovascular disease. Adams and Victors Principles of Neurology. 2007;7, 821.

7. Ong TZ, Raymond AA. Risk Factors for Stroke and Predictors of One-Month Mortality. Singapore Med J 2002;3(10):517-21.

8. Smajlovic D, Salihovic D, Ibrahimagić OC, Sinanovic O, Vidovi M. Analysis of risk factors, localization and 30-day prognosis of intracerebral hemorrhage. Bosnian journal of basic medical sciences. 2008;8(2):121-5.

9. Tetri S, Juvela S, Saloheimo P, Pyhtinen J, Hillbom M. Hypertension and diabetes as predictors of early death after spontaneous intracerebral hemorrhage. J Neurosurg. 2009;110:411-7.

10. Sessa M. Intracerebral hemorrhage and hypertension. Neurol Sci. 2008;29:S258-9.

11. Szczudlik A, Turaj W, Słowik A, Strojny. Hyperthermia is not an independent predictor of greater mortality in patients with primary intracerebral hemorrhage. J Med Sci Monit. 2002;8(10):CR702-7.

12. Boekela ET, Vroonhofb K, Huismanc A, Kampena $\mathrm{CV}$ and Kievieta WD. Clinical laboratory findings associated with in-hospital mortality. Clinica Chimica Acta. 2006;372(1-2):1-13.
13. Hitt E. Diabetes Mellitus Associated With Increased Intracerebral Hemorrhage Risk in Younger Patients. American Diabetes Association, 2007;687.

14. Schlenk F, Vajkoczy P, Sarrafzadeh A. Inpatient Hyperglycemia Following Aneurysmal Subarachnoid Hemorrhage: Relation to Cerebral Metabolism and Outcome. Neurocritical care. 2009;11:56-63.

15. Li F, Chen H, Wang Y, Yang Y, Li C, Dong Z, et al. A new prognostic model and score to predict short term outcome after intracerebral hemorrhage. Scientific Research and Essays. 2006;6(10):2063-8.

16. Sweileh WM. Predictors of in-hospital mortality after acute stroke: impact of renal dysfunctional int. J Clin Pharmacol Ther. 2008;46(12):637-43.

17. Weir CJ, Bradford APJ, Lees KR. The prognostic value of the components of the Glasgow Coma Scale following acute stroke. QJM. 2003;96(1):67-74.

18. Md. Shahrier Mahboob et al. GCS following Ac. Stroke and in hospital outcome; Journal of medicine. 2009;10(supplement-1)11-14.

19. El-Sheikh WM. Stroke mortality: predictive value of simple laboratory tests and apache iii scoring system. Menoufiya Medical Journal 353 Stroke Mortality: Predictive, 2009;22(1):489-95.

20. Kim K. Predictors of 30-Day Mortality and 90-Day Functional Recovery after Primary Intracerebral Hemorrhage : Hospital Based Multivariate Analysis in 585 Patients. J Korean Neurosurg Soc. 2009;45:341-9.

21. Sico JJ, Myers LJ, Ordin D, Williams LS, Bravata DM. The association between hematocrit and mortality among ischemic stroke patients. Stroke. 2012;43-A, 147.

22. Woo E, Chan YW, Yu YL, Huang CY. Admission glucose level in relation to mortality and morbidity outcome in 252 stroke patients. Stroke. 1988;19:18591.

23. Bhalla A, Gupta OP, Gupta SB. Predicting mortality in stroke. Neurol India. 2002;50:279-81.

Cite this article as: Chaurasia AK, Mathur MK, Dwivedi NC, Mishra M. Stroke mortality: predictive value of simple laboratory tests and acute physiology, age, chronic health evaluation III scoring system: a hospital based study. Int J Res Med Sci 2016;4:1496-500. 